\title{
MEROMORPHIC FUNCTIONS WHICH CLUSTER ON THE BOUNDARY
}

\section{MICHAEL R. CULLEN}

Let $\psi(z)$ be a univalent meromorphic function defined on $U$, the unit disc, and let $S^{2}$ denote the Riemann sphere, that is, the one point compactification of the complex plane $R^{2}$. Let $C\left(\psi, e^{i t}\right)$ be the set of all points $\zeta$ in $S^{2}$ such that there exists a sequence $z_{n} \rightarrow e^{i t}$ with $\psi\left(z_{n}\right)$ clustering at $\zeta$. It is easily seen that $C\left(\psi, e^{i t}\right) \subset \partial \psi(U)$, and furthermore, $\partial \psi(U)$ has at least two points. If $B(z)$ is a finite Blaschke product, and $f(z)=\psi(B(z))$, then $C\left(f, e^{i t}\right) \subset \partial f(U)$. If $B(z)$ is an infinite Blaschke product, this property is destroyed since $\psi(0) \in C\left(f, e^{i t}\right)$, where the zeros of $B(z)$ cluster at $e^{i t}$.

The purpose of this paper is to present the following theorem, characterizing those meromorphic functions "clustering on the boundary" :

TheOREM. Let $f(z)$ be meromorphic in $U$. Then $C\left(f, e^{i t}\right) \subset \partial f(U)$, for all $e^{i t}$, with $\partial f(U)$ consisting of at least two points, if and only if $f(z)$ $=\psi(B(z))$, where $\psi(z)$ is a univalent meromorphic function and $B(z) a$ finite Blaschke product.

Proof. We first show $f(U)$ is simply connected, that is, $K=S^{2}-f(U)$ is connected. We may assume $K$ is compact in $R^{2}$. (Let $z_{0} \in f(U), T(z)$ $=1 /\left(z-z_{0}\right)$, and $g(z)=T(f(z))$. Then $g(U)$ contains $\infty$ and $C\left(g, e^{i t}\right)$ $C \partial g(U)$. If $g(z)=\psi(B(z))$, then $f(z)=T^{-1} \circ \psi(B(z))$.) Suppose that $K$ has two different components $K_{1}$ and $K_{2}$, and let $\zeta_{i} \in \partial f(U) \cap K_{i}$, $i=1,2$. By the Zoretti Theorem [3, p. 35], there exists a Jordan curve $J$ such that $J \cap K=\varnothing$ and $J$ separates $K_{1}$ and $K_{2}$. Since $\zeta_{i} \in \partial f(U)$, there exists $e^{i t_{i}}$ such that $\zeta_{i} \in C\left(f, e^{i t_{i}}\right)$, and since this latter set is connected, it is contained in $K_{i}$. Choose sequences $z_{n} \rightarrow e^{i t_{1}}$ and $z_{n}^{\prime}$ $\rightarrow e^{i t_{2}}$ such that

(1) $\left|z_{n}\right| \nearrow 1$ and $\left|z_{n}^{\prime}\right| \nearrow 1$,

(2) $f\left(z_{n}\right)$ Einterior of $J$ and $f\left(z_{n}^{\prime}\right) \in$ exterior of $J$.

Let $\gamma_{n}$ be the circular arc $z(t)=\left|z_{n}\right| e^{i t}, t$ varying between $\arg z_{n}$ and $\arg z_{n}^{\prime}$, together with the segment $\left[z_{n}^{\prime},\left|z_{n}\right| e^{i \text { arg }} z_{n}^{\prime}\right]$. By (2), we may choose $w_{n} \in \gamma_{n}$ such that $f\left(w_{n}\right) \in J$. If $w_{n}$ clusters at $e^{i t}, f\left(w_{n}\right)$ clusters in $J$, a subset of $f(U)$, and therefore, $C\left(f, e^{i t}\right) \cap f(U) \neq \varnothing$, a contradiction. Hence $K$ is connected, and consequently $f(U)$ is simply connected.

Received by the editors April 14, 1969. 
Let $\psi$ be a 1-1 meromorphic function from $U$ onto $f(U)$ guaranteed by the Riemann mapping theorem, and consider $B(z)=\psi^{-1}(f(z))$. If $z_{n} \in U$ and $z_{n} \rightarrow e^{i t}$ with $B\left(z_{n}\right) \rightarrow w$ and $|w|<1$, then $\psi(w)=\lim _{n \rightarrow \infty} f\left(z_{n}\right)$ $\in f(U)$, contradicting the hypothesis that $C\left(f, e^{i t}\right) \subset \partial f(U)$. Hence

$$
\min _{0}\left|B\left(\mathrm{re}^{i \theta}\right)\right| \rightarrow 1 \quad \text { as } r \rightarrow 1 \text {. }
$$

Therefore, $B(z)$ has finitely many zeros, and $C\left(B, e^{i t}\right) \subset\{z:|z|=1\}$, for all $e^{i t}$. It is now easy to show that $B(z)$ is a unimodular constant times the finite Blaschke product formed by the zeros of $B(z), B_{1}(z)$, by applying the maximum modulus principle to $B / B_{1}$ and $B_{1} / B$.

CoRollary 1. If $f(z)$ is meromorphic in $U$ with $C\left(f, e^{i t}\right) \subset \partial f(U)$ for all $e^{i t}$, then,

(1) $f(z)$ is $m$-valent for some positive integer $m$;

(2) $f \in H^{p}, 0<p<1 / 2$; and

(3) $f(z)$ converges nontangentially as $z \rightarrow e^{i t}$ except possibly for a set of capacity zero.

Proof. (1) is true since $B(z)$ is $m$-valent.

(2) and (3) follow since it is well known that a univalent function possesses properties (2) and (3). (See [1, p. 473], and [2, p. 56].)

COROLlaRy 2. Let $f(z)$ be meromorphic in $U$ with $f(U)$ multiply connected. Then there exists a point $e^{i t}$ such that $C\left(f, e^{i t}\right) \cap f(U) \neq \varnothing$.

\section{BIBLIOGRAPHY}

1. G. T. Cargo, Some geometric aspects of functions of Hardy class $H^{p}$, J. Math. Anal. Appl. 7 (1963), 471-474.

2. E. F. Collingwood and A. J. Lohwater, The theory of cluster sets, Cambridge Univ. Press, New York, 1966. 1964.

3. G. T. Whyburn, Topological analysis, Princeton Univ. Press, Princeton, N. J.,

Louisiana State University 